\title{
Optimization of the incident IR heat flux upon a 3D geometry composite part (Carbon/Epoxy)
}

\author{
S. Nakouzi ${ }^{1}$, F. Berthet ${ }^{1}$, D. Delaunay ${ }^{2}$, Y. Le Maoult ${ }^{1}$, F. Schmidtt, a \\ V. Sobotka ${ }^{2, b}$ \\ ${ }^{1}$ Université de Toulouse ; Mines Albi, ICA (Institut Clément Ader) ; Campus Jarlard, F-81013 \\ Albi cedex 09, France \\ ${ }^{2}$ Université de Nantes, CNRS, Laboratoire de Thermocinétique de Nantes, UMR 6607, Le \\ Chantrerie, rue Christian Pauc, BP 50609, 44306 Nantes cedex 03, France
}

âFabrice.schmidt@mines-albi.fr, bvincent.sobotka@univ-nantes.fr

Keywords: Curing composite, Infrared oven, Radiation, Optimization, Epoxy resin, Carbon fibers.

\begin{abstract}
The main purpose of this study is to cure a 3D geometry composite part (carbon fiber reinforced epoxy matrix) using an infrared oven. The work consists in two parts. In the first part, a FE thermal model was developed, for the prediction of the infrared incident heat flux on the top surface of the composite during the curing process. This model was validated using a reference solution based on ray tracing algorithms developed in Matlab ${ }^{\circledR}$. Through the FE thermal model, an optimization study on the percentage power of each infrared heater is performed in order to optimize the incident IR heat flux uniformity on the composite. This optimization is performed using the Matlab ${ }^{\circledR}$ optimization algorithms based on Sequential Quadratic Programming and dynamically linked with the FE software COMSOL Multiphysics ${ }^{\circledR}$. In a second part, the optimized parameters set is used in a model developed for the thermo-kinetic simulations of the composite IR curing process and the predictions of the degree of cure and temperature distribution in the composite part during the curing process.
\end{abstract}

\section{Introduction}

There is growing interest in the use of composites because they are considered as potential lightweight materials for automotive parts. In a second hand rising energy costs have also promoted an interest in using infrared energy to manufacture these composites. This paper presents the work carried out on an industrial project financed by TOSHIBA LIGHTING Company that aimed to develop a new process to cure the composite using an infrared oven.

In a previous work, we presented numerical simulations $[1,2]$, based on ray tracing method, to compute the incident heat flux on the top surface of a $(140 \times 160 \times 6(\mathrm{~mm}))$ composite plate placed at the center of an IR oven composed of nine halogen lamps. This heat flux was coupled with the exothermal heat flux released during curing the epoxy resin using Finite Element simulations to predict the degree of cure and the temperature distribution in the composite. Due to the complexity of determining thermo-physical properties of the Carbon/Epoxy system, we measured thermal conductivity of the raw and cured composites based on the work of D. Lecointe [3] at LTN Nantes [4].

During the IR curing of the composites process, IR heat flux is absorbed on the top surface of the composite which has a low thermal conductivity. The absorbed heat flux (i.e heat amount) is extensively affected by the oven geometry and the nominal IR heaters' powers and by the composite geometry. Low absorbed IR heat flux value can lead to a weak degree of cure while high absorbed heat flux may cause degradation phenomena of the epoxy matrix; in both cases, bad composite qualities are obtained.

Consequently, the incident heat flux on the top surface of the composite needs to be optimized for this process application. This can be achieved either by performing an essential number of experiments, which increase the cost and the required development time of the desired product or by a parametric investigation through numerical simulation. Therefore a validated numerical simulation tool for the infrared heat flux analysis and optimization needs to be developed. 
In this work, considering the 3D shape of the composite part, only using advanced numerical tools will be possible to accomplish all the project industrial requirements. The academic algorithms based on ray tracing method $[1,2]$ and the FE commercial software based on radiosity method are two powerful simulation tools which, when operated by an expert user, can produce excellent result.

\section{Composite heating modeling}

Although ray tracing methods were used successfully previously [1,2], it is not convenient to use it in an industrial application because of the computational expense when solving the radiation problems and time-consuming resulting from coupling ray tracing method with the optimization algorithms. This is why a complete FEM model for such geometries can greatly help in an industrial study. Nevertheless, ray tracing method has been used to provide a reference solution in our simulations. The method employed by the FE heat transfer solver to compute the radiation heat transfer between opaque diffuse surfaces is based upon radiosity which is defined as the total radiative flux leaving a surface.

The radiosity method subdivides a scene into surface patches that possess Lambertian optical characteristics and subsequently determine a condition in which the light transfer between the patches constitutes an energetic equilibrium. See [5].

Composite 3D geometry is presented in figure 1; it is heated by one IR heater. We compared the results of the radiosity method implemented in COMSOL with RAYHEAT (the in-lab developed software based on ray tracing algorithms).
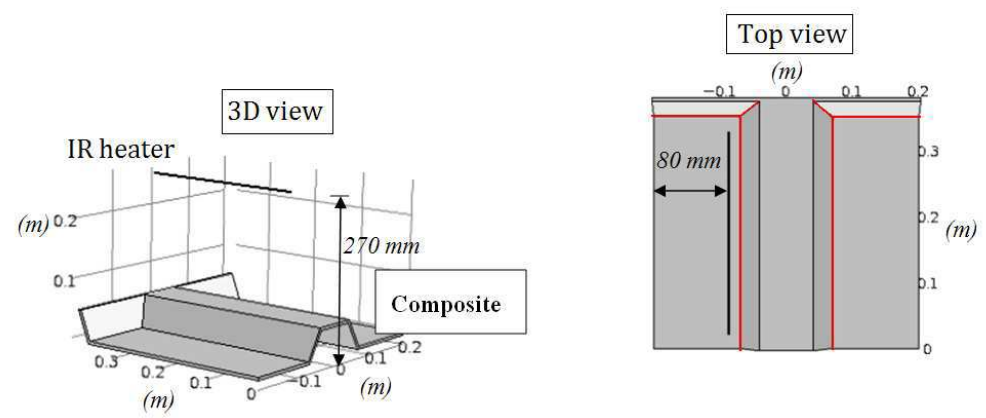

Figure 1 - Geometrical configuration of the IR heater and the composite

The composite was meshed into 9638 tetrahedral elements; a very dense mesh is used for the volume material discretization along and near the edges highlighted in figure 1 , as higher temperature gradients will occur here, while a coarser mesh is used in the remaining volume. 


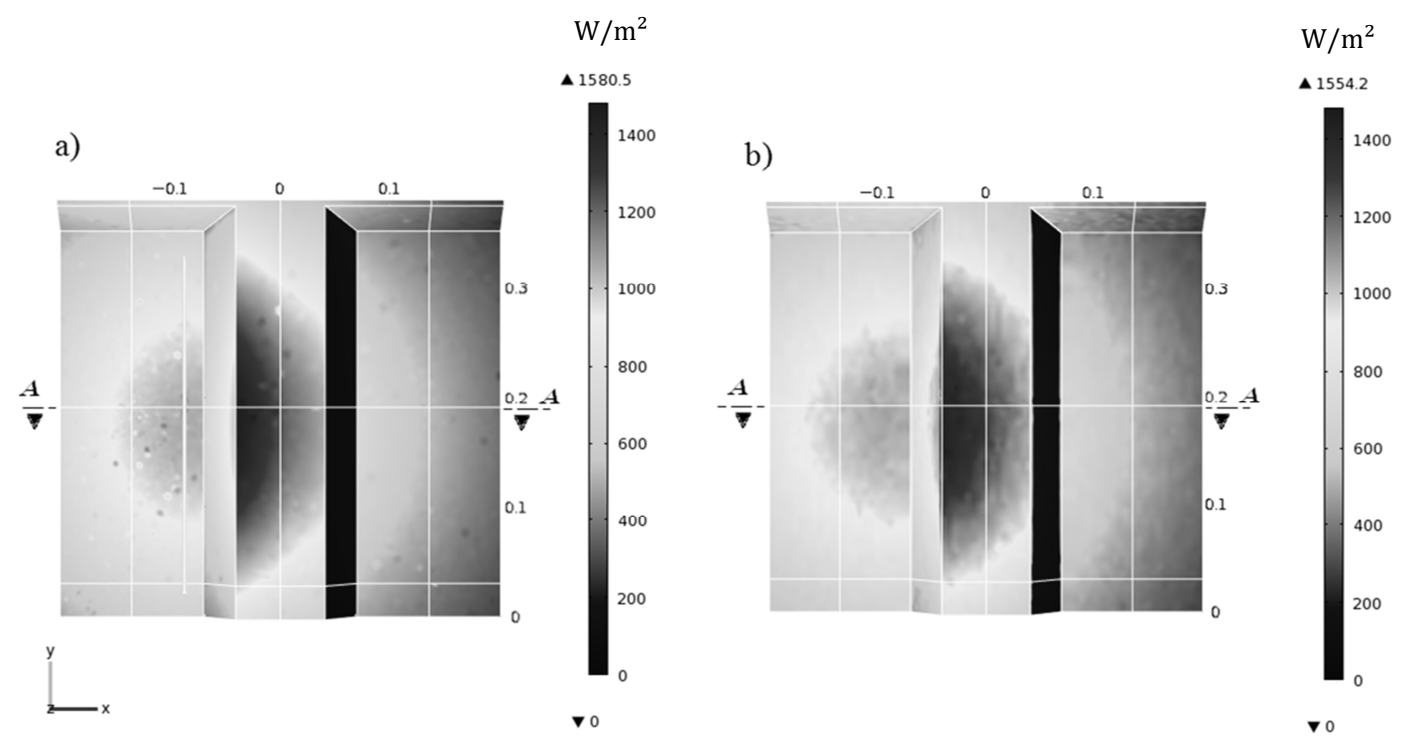

Figure 2 - Incident IR heat flux on the composite a) Radiosity method b) Ray tracing method

Note that the IR heat flux computed in Matlab using the in-house developed algorithms based on ray tracing method was exported to the COMSOL graphical interface for visualizing it on the geometry (figure $2 b$ ).

Comparing the results plotted in figure 3, the output based on FE analysis shows a very close agreement with the results based on the ray tracing algorithms. This agreement demonstrates the validity of using the FE procedures to predict the incident heat flux on the top surface of the composite.

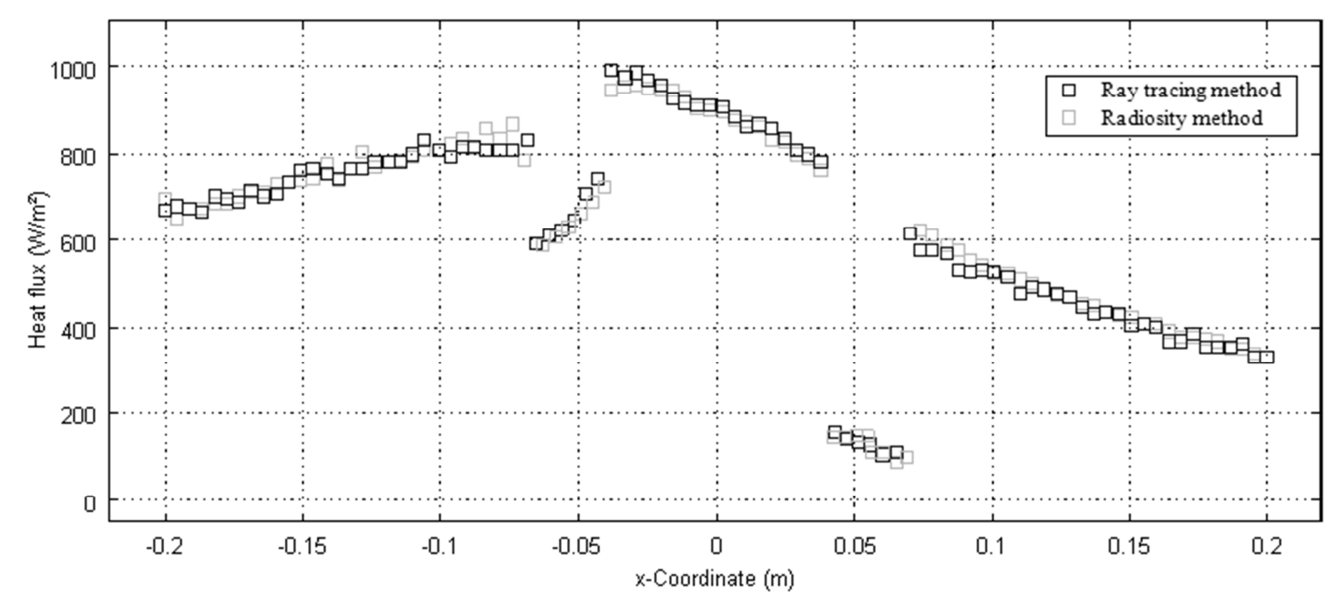

Figure 3 - Incident IR heat flux at section A-A: Ray tracing and Radiosity methods

\section{Benefits}

Compared to the ray tracing method, Radiosity method provides two significant advantages:

- Radiosity method is not restricted only for a known geometry; we can easily use it for different geometries in the industrial applications.

- It is not convenient to couple IR radiation with optimization strategy because of the CPU time of ray tracing method. 


\section{Problem Formulation of Design Optimization for IR oven}

The final mechanical properties of the composite are related to the $3 \mathrm{D}$ temperature distribution in the composite during the curing process. The successful manufacture of composites using the infrared oven can be difficult due to the exothermic nature of the resin polymerization and the low thermal conductivity of the composite [5] which affects temperature distribution in the composite.

The objective of the simulations has been to optimize the IR heat flux uniformity on the top surface of a 3D composite part (and then the composite temperature uniformity). IR oven design parameters were determined using COMSOL Multiphysics software.

Oven's geometrical configuration is similar to an industrial one; it is presented in figure 4 and is composed of 17 halogen lamps and a diffuse reflector. Note that radiative properties of the IR heaters, the diffuse reflector and the composite are listed in [6-8]. The optimal design of this oven with improved properties over the existing or preliminary design can be achieved by modifying the percentage power of each infrared heater.

By choosing the appropriate power percentage for each IR heater based on optimization techniques, the composite part is able to achieve higher mechanical properties.

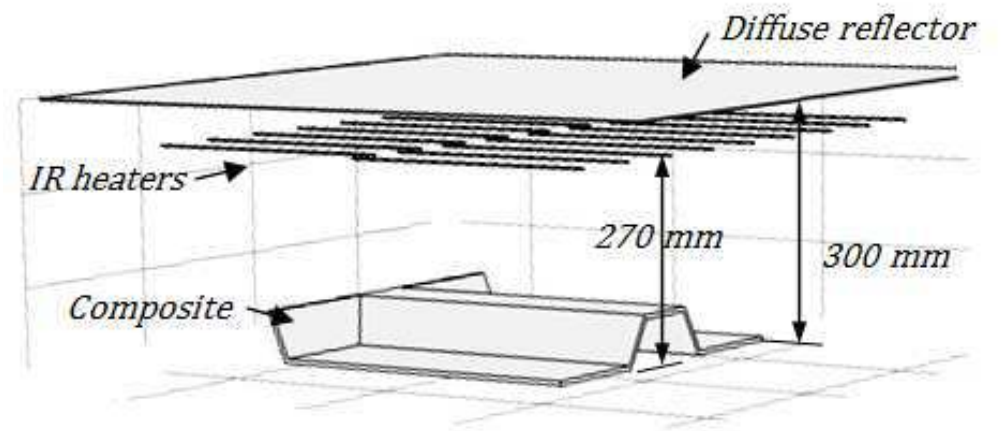

Figure 4 - IR oven geometrical configuration

\section{Optimization Problem}

Simulations were carried out close to the industrial requirements; Oven configuration is designed using the Sequential Quadratic Programming (SQP) which is a well-known algorithm to solve a non-linear programming problem [9] in which an objective function and constraints may be nonlinear functions of the design parameters. The objective function is designed so that to minimize the root mean square deviations of the computed IR heat flux on the top surface of the composite from the desired one. The optimization problem is formulated as:

$$
\begin{gathered}
\text { Minimise } J(P)=\int_{S} \frac{\left(\emptyset(x, y, z)-\emptyset_{c}\right)^{2}}{\emptyset_{c}^{2}} \\
\text { Subject to: } P_{i}^{L} \leq P \leq P_{i}^{U}
\end{gathered}
$$

Where $P=\left[P_{1}, P_{2}, \ldots, P_{17}\right]$ is the optimization variable vector corresponding to the power percentage of the IR emitters $\left(P_{i}=\frac{\text { Effective Power }}{\text { Nominal power }} * 100\right)$, Nominal power of the IR emitters is equal to $1000 \mathrm{~W}, S$ is the composite top surface, $\varnothing(x, y, z)$ is the incident heat flux on the top surface of the composite as function as the spatial coordinates $(x, y, z)$ and $\emptyset_{c}=2000 \mathrm{~W} / \mathrm{m}^{2}$ is the desired incident heat flux on the top surface of the composite. 
The convergence of objective function with respect to iteration steps is plotted in figure 5:

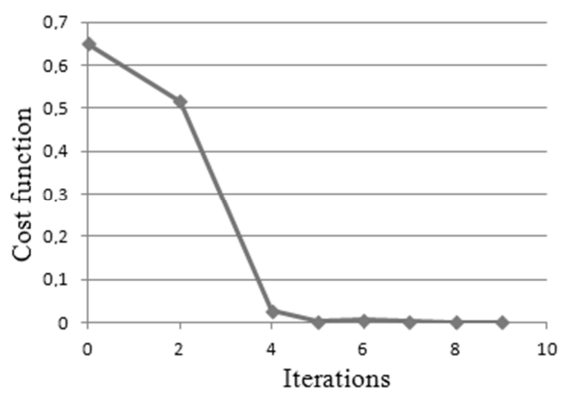

Figure 5 - Objective function value versus iteration

Figure 6 illustrates the external temperature distribution cartography of the composite before and after optimization respectively:

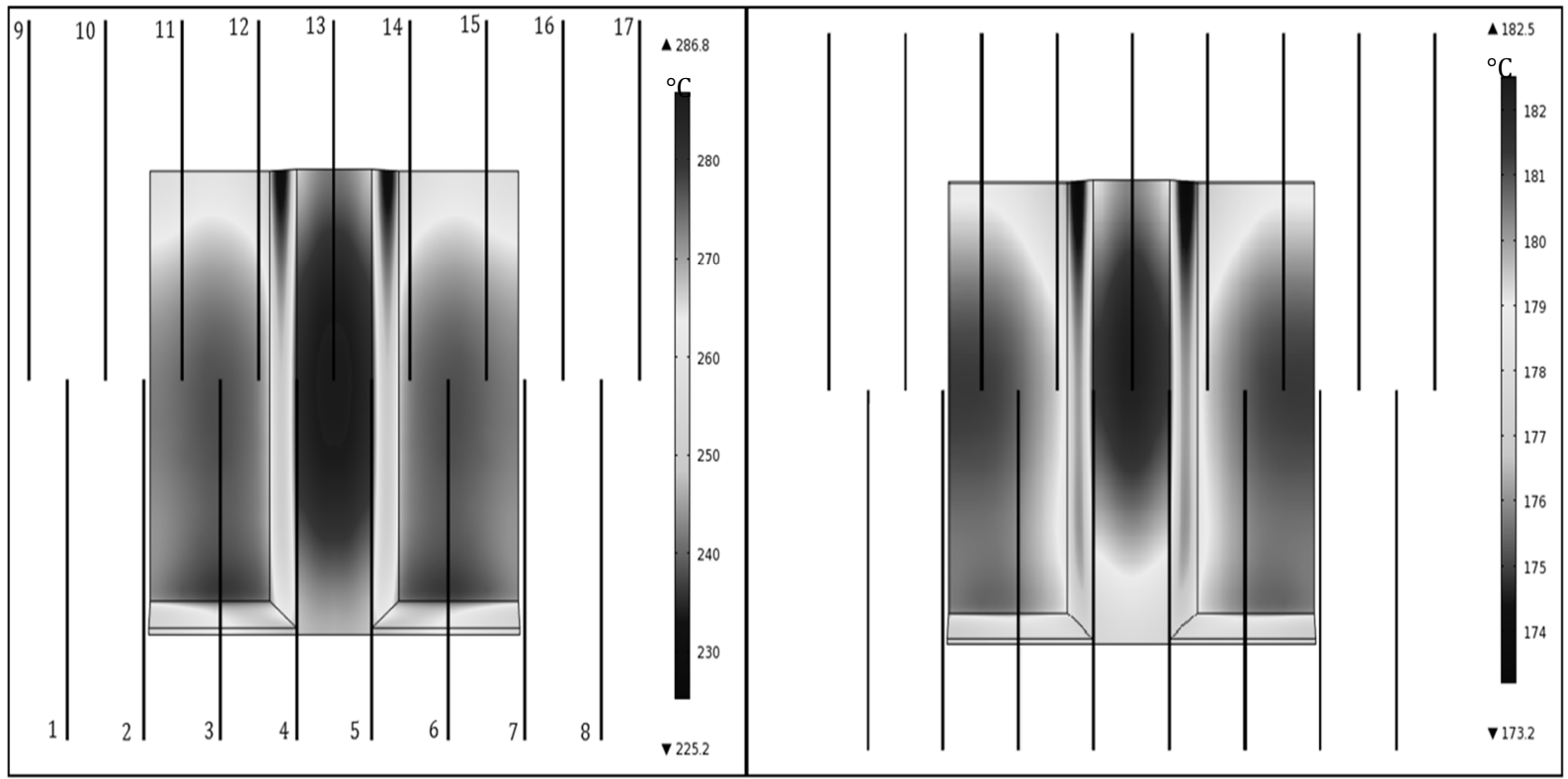

Figure 6 - External temperature $\left({ }^{\circ} \mathrm{C}\right)$ distribution cartography a) before and b) after optimization The optimized results for the IR heaters' powers numbered in figure 6 are presented in table1:

Table 1 - Optimization variables

\begin{tabular}{c|ccccccccccccccccc}
$\begin{array}{c}\text { IR Heater } \\
\mathrm{N}^{\circ}\end{array}$ & 1 & 2 & 3 & 4 & 5 & 6 & 7 & 8 & 9 & 10 & 11 & 12 & 13 & 14 & 15 & 16 & 17 \\
\hline $\begin{array}{c}\text { Initial } \\
\text { Power (\%) }\end{array}$ & 50 & 50 & 50 & 50 & 50 & 50 & 50 & 50 & 50 & 50 & 50 & 50 & 50 & 50 & 50 & 50 & 50 \\
\hline $\begin{array}{c}\text { Final } \\
\text { Power (\%) }\end{array}$ & 25 & 21 & 16 & 14 & 12 & 17 & 19 & 26 & 24 & 31 & 25 & 19 & 14 & 15 & 20 & 29 & 26
\end{tabular}

\section{Conclusion}

An efficient methodology for the simulations of an IR composite curing process has been presented in this paper. Initially, a transient thermo-kinetic FE-model is developed and used to cure the composite. In a second hand, IR heat flux distribution on the top surface of the composite was computed and integrated with optimization algorithms to perform explicit IR oven design analysis and optimization. This optimization problem is solved by a sequential quadratic programming algorithm implemented in Matlab ${ }^{\circledR}$. 
The combined FE method and optimization analysis presented in this study can be used effectively in the IR oven's design application. The proposed methodology is quite flexible; it can be modified properly and used for any composite geometry. It offers the capability of providing a reliable estimation of the IR oven design parameters set, especially the power percentage of each IR heater to adequately cure the composite.

\section{Acknowledgments}

Financial support for this work was provided by TOSHIBA LIGHTING (France) Company. The authors are grateful to Mr. D. Goyot for his support and his attention to this work.

\section{References}

[1] S. Nakouzi, J. Pancrace, F. Schmidt, Y. Le MAoult, F. Berthet. "Curing Simulation of Composites Coupled with Infrared heating.» International Journal of Material Forming, 2010: 587-590.

[2] S. Nakouzi, J. Pancrace, F. Schmidt, Y. Le MAoult, F. Berthet. "Simulations of an Infrared Composite Curing process." Advanced Engineering Materials, 2011: 604-608.

[3] D. Lecointe. Caractérisation et simulation des processus de transferts lors d'injection de résine pour le procédé RTM. Thèse de Doctorat: Ecole doctorale science pour l'ingénieur de Nantes, 1999.

[4] J.-L. Bailleul, D. Delaunay, Y. Jarny, T. Jurkowski. «Thermal Conductivity of Unidirectional Reinforced Composite Materials-Experimental Measurement as a Function of State of Cure.» Journal of Reinforced Plastics and Composites, 2001: 20-52.

[5] R. Siegel,J.R. Howell . Thermal Radiation Heat Transfer. Washington DC: Hemisphere Publishing.

[6] B. Cosson, F. Schmidt, Y. Le Maoult, M. Bordival. «Infrared heating stage simulation of semi-transparent media (PET) using ray tracing method.» International Journal of Material Forming, 2010.

[7] B-C. Chern, T.J. Moon, J.R Howell. «On-Line Processing of Unidirectional Fiber Composites Using Radiative Heating: I. Model.» Journal of Composite Materials, 2002: 1905.

[8] B-C. Chern, T.J. Moon, J.R Howell. «On-line Processing of Unidirectional Fiber Composites Using Radiative Heating: II. Radiative Properties, Experimental Validation and Process Parameter Selection.» Journal of Composite Materials, 2002: 1935.

[9] COMSOL Multiphysics. «Heat Transfer Module User's Guide.» 